brain imaging in 78 patients examined (MRI 45, CT 27, both 6) were not specified in 17, atypical headache pattern in 12 , parental concern 12 , physician concern about cerebral tumor 11, systemic symptoms of fatigue and weight loss in 11 , focal symptoms or signs during headaches in 7 , neurologic or ocular abnormalities 6 , and increasing severity or frequency of headaches in 5 . None of the scans showed brain tumor, vascular abnormality, or hydrocephalus that required neurosurgical intervention. Abnormal scans in 11 patients included evidence of chronic sinusitis in 7 , a neuroepithelial cyst adjacent to the foramen of Monroe treated conservatively in 1, right temporal arachnoid cyst in 1, left cerebral hemiatrophy in 1, and Dandy-Walker malformation in 1. (Maytal J et al. The value of brain imaging in children with headaches. Pediatrics September 1995;96:413-416). (Reprints: Joseph Maytal MD, Division of Pediatric Neurology, Schneider Children's Hospital, Long Island Jewish Medical Center, New Hyde Park, NY 11040).

COMMENT. The authors conclude that brain imaging has very limited value in the management of childhood headaches in the absence of clinical signs of structural brain lesion. Although none of 11 (14\%) positive scans was considered indicative of a treatable disease at the time, the abnormalities uncovered might potentially have proved significant. I would agree that brain imaging is not warranted in patients with well-defined migraine and that routine use of neuroimaging is to be discouraged. However, patients referred to a pediatric neurologist for chronic headache have usually been screened by their pediatrician or family physician and the pressures for neuroimaging may be more demanding.

An MRI should be considered especially in those with atypical recurrent headaches, a recent change in the character of the headache, persistent vomiting, a history of seizures, abnormal neurologic findings, and in younger age groups. However, the heavy sedation required for the MRI in a young child and risks of an adverse reaction to contrast medium with CT must be weighed against the benefits of the study. Headache as the sole manifestation of brain tumor is a rare occurrence. As a further consideration, the luxury of follow-up evaluation and observation over time may not be available to the neurologist who examines a patient in consultation, and the deferral of imaging may not be practical or judicious. For further commentary on the indications for imaging in headache and the value of an EEG as a preliminary test in diagnosis, see Progress in Pediatric Neurology II, PNB Publishers, 1994, pp164-6).

\title{
TRANSIENT HEADACHE AND CSF LYMPHOCYTOSIS
}

A transient syndrome of migrainous headache with neurologic deficits and CSF lymphocytic pleocytosis is described in 7 young adult patients examined at the Strong Memorial Hospital, University of Rochester Medical Center, Rochester, NY. The clinical characteristics of 33 similar cases, 13 in children and adolescents, previously reported in the literature are also analysed. The diagnostic criteria for this syndrome include severe headache, temporary (<4 days) neurologic deficit, CSF lymphocytosis $(16-350 \mathrm{WBC} / \mathrm{mm} 3)$, and self-limited course (range 1-84 days, mean 21 days). The neurologic signs and symptoms were usually transient hemiparesis or sensory changes, confusional episodes, and aphasia. CSF protein was increased in $91 \%$ of cases, CSF pressure increased in 73\%, focal, nonepileptiform EEG irregularities were 
recorded in $72 \%$, and a viral illness or fever preceded the headaches in $50 \%$. Apart from 2 patients showing unidentified bright objects on MRI, neuroimaging studies were negative. The headaches had characteristics of migraine with aura, unilateral location, pulsating quality, nausea, and photophobia, but unlike typical migraine, the headache syndrome was not chronic. The syndrome resolved within 3 months. The cause is undetermined. (Berg MJ, Williams LS. The transient syndrome of headache with neurologic deficits and CSF lymphocytosis. Neurology September 1995;45:1648-1654). (Reprints: Dr Michel J Berg, Box 673, Department of Neurology, University of Rochester Medical Center, 60 Elmwood Avenue, Rochester, NY 14642).

COMMENT. The differential diagnoses listed by the authors for this syndrome include Lyme neuroborreliosis, neurosyphilis, neurobrucellosis, mycoplasma infection, granulomatous meningitis, neoplastic meningitis, autoimmune disease, HIV meningitis, and a side effect of cerebral angiography. Other illnesses with similar features are hemiplegic migraine, seizure disorder, and recurrent aseptic

meningitis (Mollaret's meningitis). The syndrome is self limited, and a viral etiology seems likely.

\section{NEONATAL ENCEPHALOPATHY}

\section{ANTEPARTUM CAUSES OF ENCEPHALOPATHY}

Adverse factors in the family and maternal history, pregnancy, and birth related to the occurrence of neonatal encephalopathy (NE) in full term newborn infants were evaluated in a matched case-control study at the Institute for Child Health Research, West Perth, and the Department of Neonatology, Princess Margaret Hospital for Children, Subiacco, Western Australia. Of 89 cases studied, 42 met criteria for moderate or severe neonatal encephalopathy: severe NE -mechanical ventilation required for $>24$ hours, multiple anticonvulsants, coma, or death; moderate NE: -neurologic abnormalities or seizures requiring anticonvulsants, but resolving before discharge. The estimated incidence of NE in the first week of life was 3.75 per 1000 full term live births, and a case fatality of $8 \%$. Intrapartum hypoxia was the cause of NE in only 5 cases, and antepartum factors were more significant and frequent. Maternal vaginal bleeding in pregnancy, physical trauma during pregnancy, maternal thyroxine treatment, and congenital abnormalities were significantly more frequent in NE patients than in controls. Maternal alcohol consumption, smoking during pregnancy, and gestational diabetes were not related to NE. (Adamson SJ et al. Predictors of neonatal encephalopathy in full term infants. BMI 2 September 1995;311:598602). (Respond: Professor Fiona Stanley, Institute for Child Health Research, PO Box 855, West Perth 6872, Western Australia, Australia).

COMMENT. Antepartum factors and preexisting neurologic abnormalities are important in the cause of neonatal encephalopathy occurring in full term infants. Intrapartum hypoxia is significant in only $6 \%$ of cases.

Problems with definitions and classifications of newborn encephalopathy are reviewed in Progress in Pediatric Neurology II, 1994, pp321-2. The clinical features of hypoxic-ischemic encephalopathy are not specific, and similar symptoms may be caused by metabolic disorders, infection or cerebral malformations. 